20

\title{
Температурная эволюция диэлектрического отклика $\alpha$-лактозы моногидрата в терагерцовом диапазоне частот
}

\author{
(С Г.А. Командин ${ }^{1}$, О.Е. Породинков ${ }^{1}$, В.С. Ноздрин ${ }^{1}$, Г.Р. Мусина ${ }^{1,2}$, Н.В. Черномырдин ${ }^{1,2}$, \\ К.И. Зайцев ${ }^{1,2}$, И.Е. Спектор ${ }^{1}$ \\ ${ }^{1}$ Институт общей фризики им. А.М. Прохорова РАН, \\ 119991 Москва, Россия \\ ${ }^{2}$ Московский государственный технический университет им. Н.Э. Баумана, \\ 105005 Москва,Россия \\ e-mail: gakomandin@mail.ru
}

Поступила в редакцию 31.12.2019 г.

В окончательной редакции 10.01.2020 г.

Принята к публикации 28.02.2020 г.

\begin{abstract}
Проведены измерения спектров пропускания спрессованных микрокристаллических образцов $\alpha$-лактозы моногидрата с помощью терагерцовой импульсной спектроскопии в температурном диапазоне существования твердой фазы $10-475 \mathrm{~K}$. Анализ наблюдаемых линий поглощения с применением модели классического осциллятора позволил выявить сложную температурную эволюцию собственных частот резонансов, а также определить область существования фазы отклика $\alpha$-лактозы моногидрата. Полученные данные могут найти практическое применение в различных областях терагерцовой оптики, включая фармакологию, пищевую индустрию, аналитическую химию и биофотонику.
\end{abstract}

Ключевые слова: терагерцовая импульсная спектроскопия, микрокристаллические органические соединения, дисахариды, $\alpha$-лактоза моногидрат, диэлектрический отклик, необратимые фазовые переходы.

DOI: $10.21883 /$ OS.2020.06.49406.20-20

\section{Введение}

Температурная стабильность полиморфных форм органических соединений является критически важным фактором для многих задач фармакологии, пищевой индустрии и биологии. Среди многочисленных методов контроля молекулярной и кристаллической структуры терагерцовая (ТГц) спектроскопия $[1,2]$ в настоящее время еще не имеет столь же широкого распространения как спектроскопия комбинационного рассеяния света (КРС) и инфракрасная (ИК) спектроскопия, несмотря на ее большой потенциал. К преимуществам данного метода следует отнести низкую ионизирующую способность ТГц излучения и незначительное рассеяние ТГц излучения микрокристаллическими образцами $[3,4]$. Аппаратура ТГц импульсной спектроскопии не требует применения высокого напряжения или сильных магнитных полей и может быть выполнена в компактном и доступном варианте $[5,6]$. Хотя частотный диапазон, перекрываемый ТГц импульсной спектроскопией, меньше чем для традиционных методов ИК спектроскопии и КРС, этот метод является самодостаточным или может применяться в качестве дополнительного аналитического инструмента. Методы ТГц импульсной спектроскопии принципиально позволяют проводить in situ контроль качества материала (или препарата) непосредственно в ходе технологического цикла производства $[7,8]$.

Среди многообразия органических соединений монои дисахариды являются необычайно удобными материалами для контроля их характеристик методами ТГц диэлектрической спектроскопии, что определяется наличием в ТГц диапазоне высокодобротных линий поглощения. Анализ эволюции параметров этих линий позволяет контролировать структурные изменения в образцах. Отмеченные соединения нашли широкое практическое применение. В частности, их водные растворы используются в качестве гиперосмотических агентов для иммерсионного оптического просветления тканей в видимом, инфракрасном и ТГц диапазонах $[9,10]$.

Процессы гидратации и дегидратации лактозы активно изучаются с использованием различных подходов, включая методы ТГц импульсной спектроскопии [11-14], поскольку они чувствительны к влагонасыщенности пористых сред [15]. Изучению трансформации молекулярной структуры органических кристаллов методами ТГц спектроскопии также посвящен ряд работ $[16,17]$, из которых, в частности, следует, что методы ТГц спектроскопии обладают достаточной чувствительностью к регистрации трансформации колебательного спектра при изменениях молекулярной структуры.

Одним из широко распространенных дисахаридов является $\alpha$-лактоза моногидрат ( $\alpha$-LM). На рис. 1 показана структура базиса монокристалла $\alpha$-LM, схематично представленная по данным рентгеноструктурных исследований, опубликованных в работах $[18,19]$.

Дисахарид (4-O- $\beta-\mathrm{D}-$ galactopyranosil $-\alpha-\mathrm{D}-$ glucopyranosemonohydrate $) \quad 2 \cdot\left(\mathrm{C}_{12} \mathrm{H}_{22} \mathrm{O}_{11} \cdot \mathrm{H}_{2} \mathrm{O}\right)-\alpha-\mathrm{LM}$ состоит из двух моносахаридов - глюкозы и галактозы, объединенных гликозидной связью. В $\alpha$-форме две 


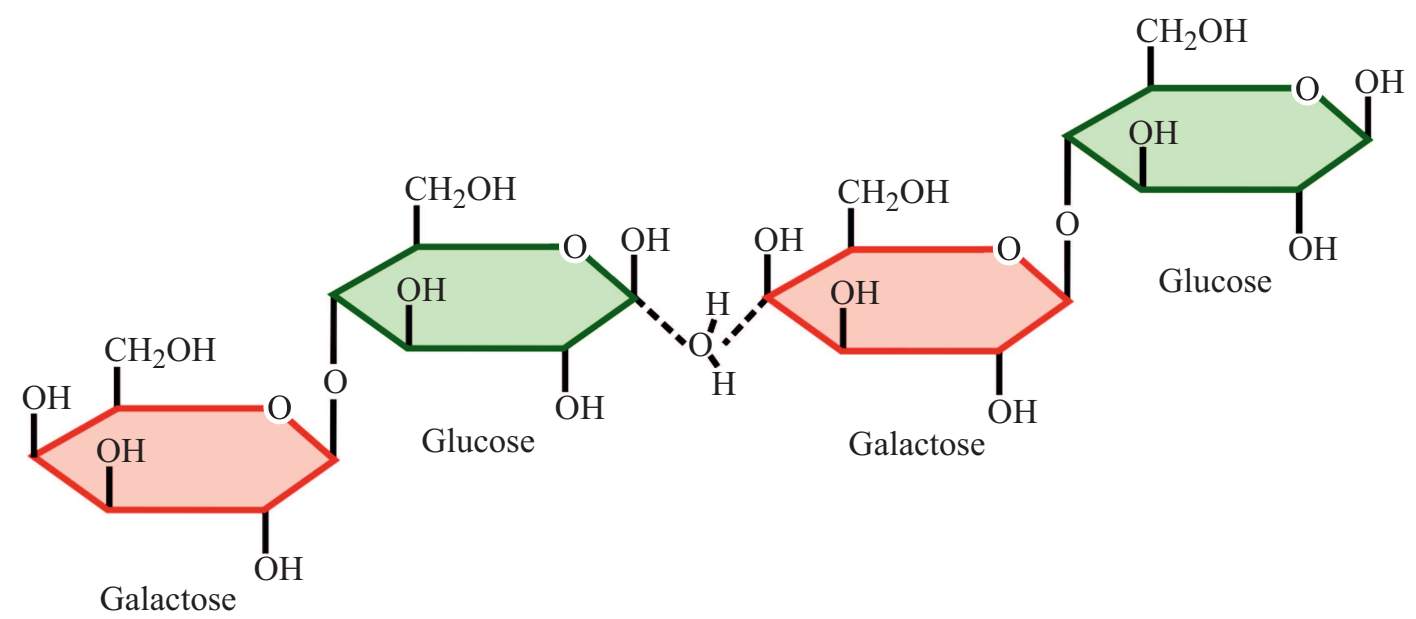

Рис. 1. Структура базиса кристалла $\alpha$-лактозы моногидрата ( $\alpha$-LM) по данным $[11,12]$.

молекулы лактозы связаны через молекулу $\mathrm{H}_{2} \mathrm{O}$, как показано на рис. 1. Наличие такой связи определяет узкий резонанс на частоте $17.6 \mathrm{~cm}^{-1}$ при комнатной температуре, в то время как в спектрах безводной $\beta$-формы лактозы этот резонанс не наблюдается [20].

Известно также наличие пиков поглощения на более высоких частотах [21]. В целом спектр электродипольных возбуждений $\alpha$-LM при комнатной температуре может быть описан суммой высокодобротных резонансных линий, уширенных полос поглощения и квазирелаксационного фона [22].

В спектре $\alpha$-LM есть, как минимум, две асимметричные полосы выше $50 \mathrm{~cm}^{-1}$. Для выяснения структуры этих полос необходимы низкотемпературные измерения спектров пропускания $\alpha$-LM, так как при понижении температуры константы затухания полос поглощения уменьшаются, что позволяет разрешить близкорасположенные резонансы с перекрывающимися контурами. При нагреве $\alpha$-LM выше комнатной температуры изменение спектра может происходить не только за счет увеличения констант затуханий резонансов, но также и вследствие изменений молекулярной и кристаллической структур. Именно поэтому целью работы является изучение температурных преобразований в спектрах диэлектрического отклика $\alpha$-LM методами ТГц импульсной спектроскопии и на их основе выявление трансформаций молекулярной структуры этого соединения. Для оценки границ температурной стабильности и наличия структурных переходов $\alpha$-LM в работе рассматривается температурный диапазон от $10 \mathrm{~K}$ вплоть до температуры плавления $-T_{\text {melt }}=476 \mathrm{~K}$.

\section{Экспериментальное исследование и моделирование спектров}

Для изготовления образцов использован микрокристаллический порошок $\alpha$-лактозы моногидрата SuperTab $^{\circledR} \quad$ 30GRDFEpharma (Германия). Из этого порошка изготовлены плоскопараллельные образцы диаметром $13 \mathrm{~mm}$. Образцы спрессованы гидравлическим прессом LabTools (Россия) в стандартной откачиваемой пресс-форме „Sресас“ (Англия). Усилие прессования составило $20-30 \mathrm{kN}$. Образцы глюкозы были изготовлены прессованием по такой же методике, как и образцы лактозы. Для приготовления образцов использована кристаллическая глюкоза производства „Hungrana KFT“ (Венгрия). Спектры пропускания и фазового сдвига в ТГц диапазоне измерены на лабораторном спектрометре с временным разрешением (TDS) $[5,6,22]$.

Следует отметить, что многие органические соединения, в частности сахара, при нагреве могут взаимодействовать с содержащимися в атмосфере азотом и кислородом. Для исключения таких реакций все измерения выполнены при низком остаточном давлении (не более $\left.10^{-2} \mathrm{mbar}\right)$ в рабочем объеме криостата и термостата. Это исключает не только изменение химического состава лактозы, но и влияние на получаемые спектры узких резонансных линий водяного пара атмосферы. Измерения выполнены по стандартной процедуре измерения фототока в приемной антенне спектрометра с образцом и без образца в ТГц тракте спектрометра. Далее с применением фурье-преобразования временных зависимостей фототока были рассчитаны спектры пропускания и фазового сдвига в образце. Измерения при низких температурах (от 300 до $10 \mathrm{~K}$ ) выполнены в оптическом криостате, описание которого приведено в [6]. Измерения спектров пропускания в диапазоне температур от 300 до $475 \mathrm{~K}$ проведены в термостате. Поскольку давление в оптической камере спектрометра было таким же, как и в объеме печки, в данном эксперименте мы не использовали окна, поскольку их нагрев мог бы привести к изменениям в спектрах.

На низкочастотном краю диапазона мы дополнительно провели калибровочные измерения образцов лактозы с использованием когерентного спектрометра с квазимо- 


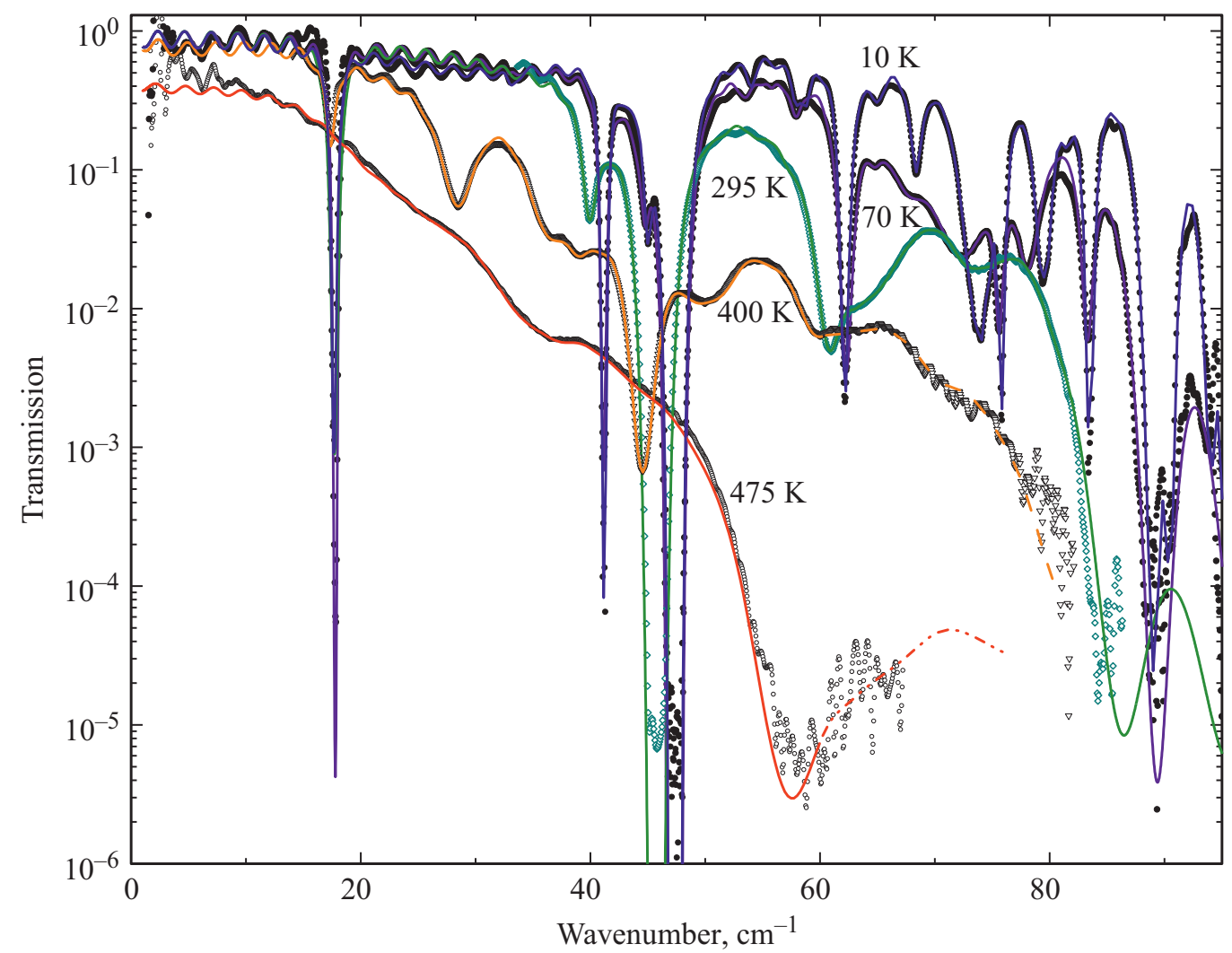

Рис. 2. Спектры пропускания $\alpha$-LM при различных температурах: точки - экспериментальные данные, сплошные линии моделирование полос поглощения с использованием выражения (1).

нохроматическим перестраиваемым по частоте генератором на основе ламп обратной волны (ЛОВ) [23].

Для моделирования спектров диэлектрического отклика в виде частотных зависимостей комплексной диэлектрической проницаемости мы использовали простую модель классического осциллятора:

$$
\varepsilon(v)=\varepsilon_{\infty}+\sum_{j=1}^{N} \frac{\Delta \varepsilon_{j} v_{j}}{v_{j}^{2}-v^{2}+i v \gamma_{j}} .
$$

Спектры действительной и мнимой частей комплексной диэлектрической проницаемости $\varepsilon(v)=\varepsilon(v)+i \varepsilon^{\prime}(v)$ рассчитываются путем подбора параметров модели диэлектрического вклада моды $\Delta \varepsilon_{j}$, ее частоты $v_{j}$ и константы затухания $\gamma_{j}$, при этом константа $\varepsilon_{\infty}$ включает в себя вклады всех высокочастотных процессов. Суммирование проводится по всем $N$ осцилляторам. Параметры подбираются таким образом, чтобы минимизировать среднеквадратичное отклонение расчетного спектра пропускания от экспериментальных данных.

\section{Результаты}

Экспериментальные данные получены на ТГц импульсном спектрометре с шагом по температуре $10 \mathrm{~K}$.
На рис. 2 показана выборка экспериментальных спектров, полученных при различных температурах в диапазоне от 10 до $475 \mathrm{~K}$. Спектр пропускания $\alpha$-LM при комнатной температуре схож с известными литературными данными [24,25]. Отметим, что две асимметричные полосы в области $60-75 \mathrm{~cm}^{-1}$, наблюдаемые при комнатной температуре, разделяются на узкие резонансные линии при охлаждении. Изменение температуры приводит не только к изменению ширины линии, но также и к существенному смещению их резонансных частот.

Для перекрывающихся уширенных контуров можно использовать модель, учитывающую взаимодействие мод, например модель двух взаимодействующих гармонических осцилляторов, система уравнений движения которых имеет вид [26]

$$
\left\{\begin{array}{l}
\frac{\partial^{2} y_{1}}{\partial^{2} t}+\Gamma \frac{\partial y_{1}}{\partial t}+\left(k_{1}+k_{12}\right) y_{1}-k_{12} y_{2}=e_{1} E \\
\frac{\partial^{2} y_{2}}{\partial^{2} t}+\Gamma \frac{\partial y_{2}}{\partial t}+\left(k_{2}+k_{12}\right) y_{2}-k_{12} y_{1}=e_{2} E
\end{array}\right.
$$

где $y-$ координата, $k-$ возвращающая сила, $\Gamma-$ коэффициент затухания, $e-$ эффективный заряд и $E-$ напряженность электрического поля. Для описания взаимодействия трех и более осцилляторов потребуется введение большего числа констант взаимодействия, однозначный выбор которых представляется затруднительным. 

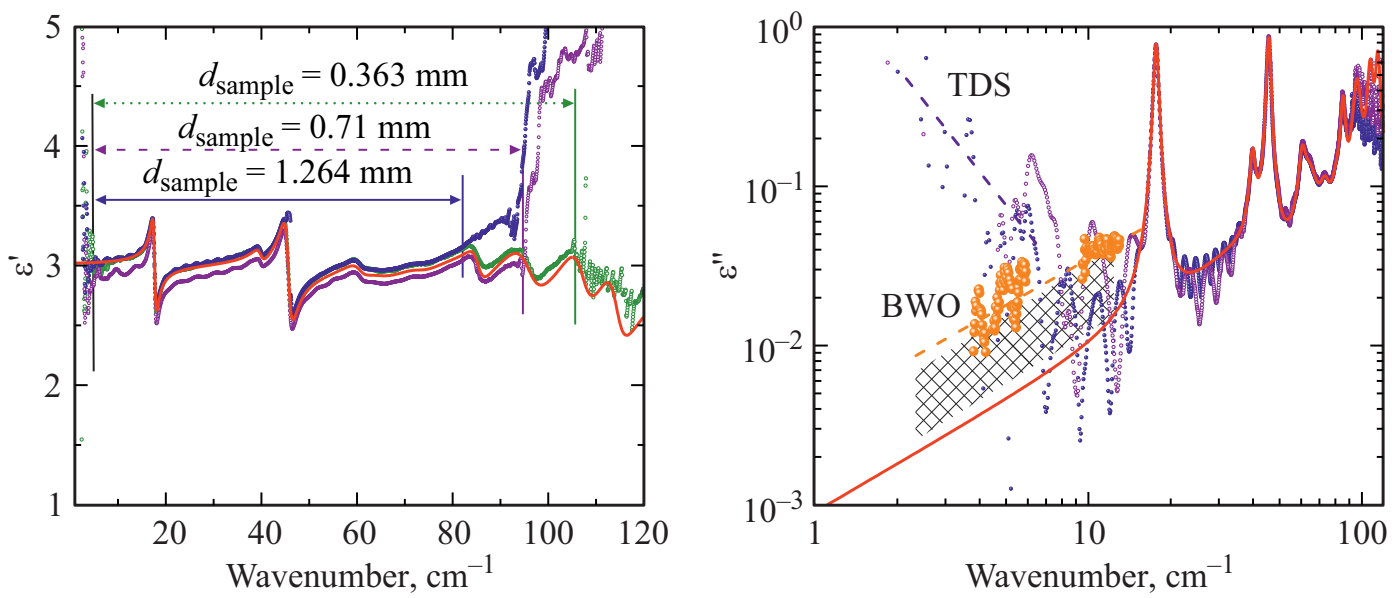

Рис. 3. Спектры действительной и мнимой частей комплексной диэлектрической проницаемости $\alpha$-LM, полученные при комнатной температуре для образцов разных толщин $(d)$ : точки - прямой расчет с применением методов решения обратных задач ТГц импульсной спектроскопии; крупные символы - данные спектроскопии на базе ЛОВ; сплошная линия - оценка диэлектрической проницаемости с применением модели классического осциллятора. Штриховые линии даны для наглядности.

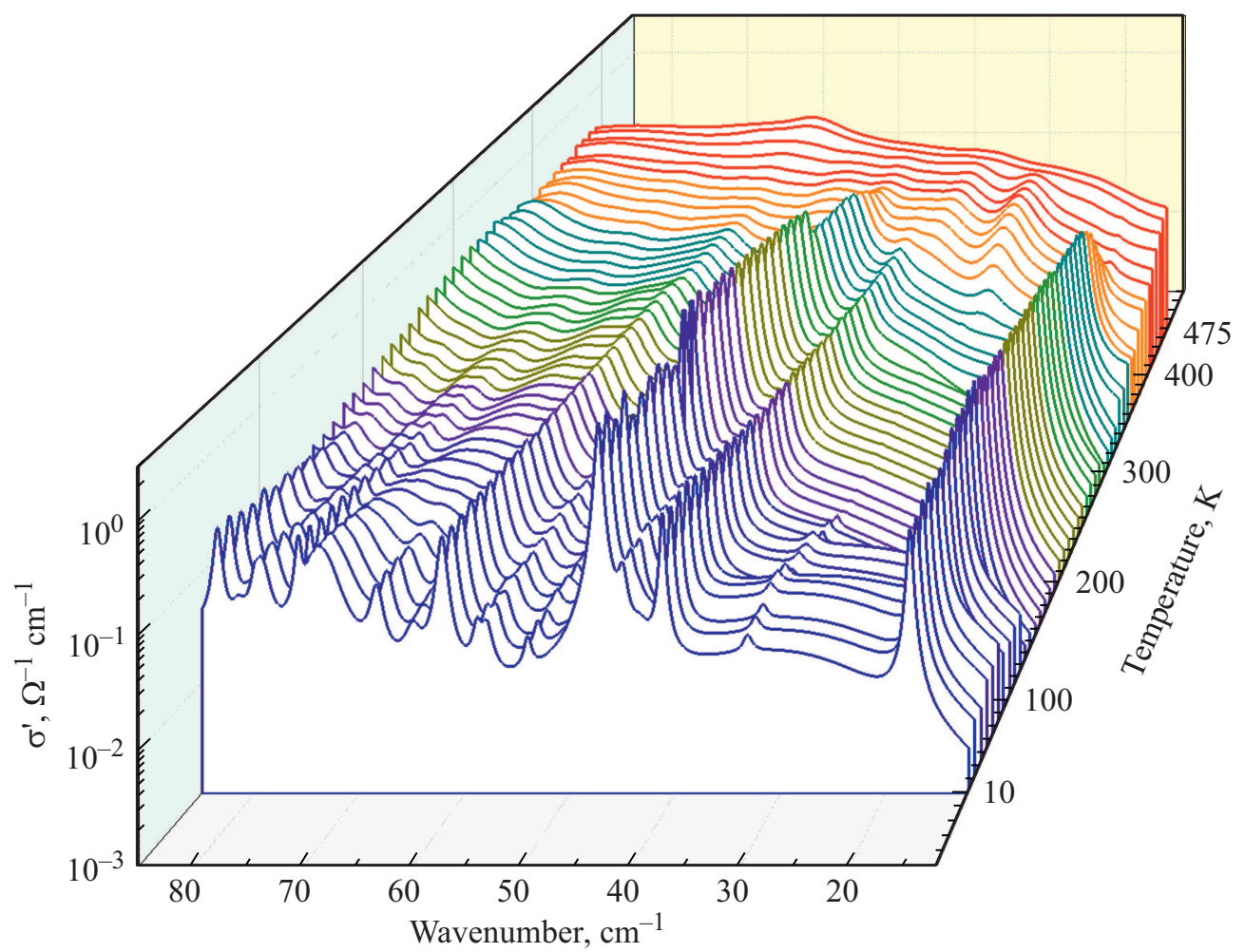

Рис. 4. Температурная эволюция диэлектрического отклика $\alpha$-LM в виде спектров динамической проводимости $\sigma(v)$.

Общий вид спектров комплексной диэлектрической проницаемости приведен на рис. 3. Отметим, что ниже $10 \mathrm{~cm}^{-1}$ спектр мнимой части, полученный по измеренным спектрам пропускания и фазы, растет с понижением частоты. Общая тенденция роста показана для наглядности штриховой линией. Такой тип дисперсии может быть связан с высокочастотным крылом контура релаксации. Для проверки мы провели измерения на этих частотах с применением спектрометра на базе ламп обратной волны. Для этого мы изготовили образец толщиной $3.5 \mathrm{~mm}$, при которой точность определения мнимой части диэлектрической проницаемости обеспечивает получение достоверных результатов.

Как видно из данных измерений с помощью ЛОВ, рост функции $\varepsilon^{\prime \prime}(v)$ не наблюдается, хотя реально измеренные диэлектрические потери на порядок больше ожидаемых из моделирования классическим осциллятором. Этот результат можно считать методическим, поскольку 

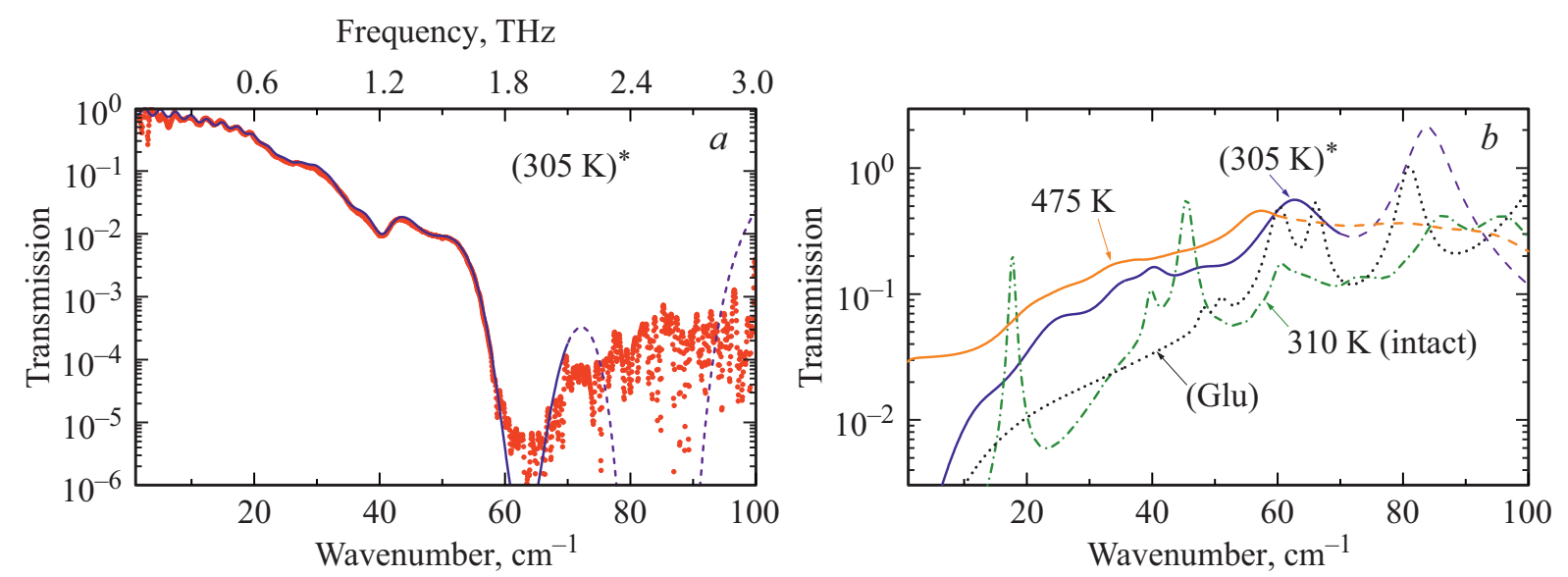

Рис. 5. (a) Спектр пропускания $\alpha$-LM после охлаждения от максимальной температуры нагрева. Точки - эксперимент, сплошная линия - моделирование, пунктирная линия - дисперсия коэффициента пропускания, смоделированная осциллятором в области низкой достоверности данных. $(b)$ Сопоставление спектров оптической проводимости исходного образца $(\alpha$-LM) до нагрева $310 \mathrm{~K}$ (штрихпунктирная линия), для максимально нагретого образца $T=475 \mathrm{~K}$ и после его охлаждения до температуры $305 \mathrm{~K}-$ сплошная линия отмечена $(305 \mathrm{~K})^{*}$. Точками показан спектр глюкозы $(\mathrm{Glu})$ при $T=300 \mathrm{~K}$.

он определяет низкочастотную границу применимости данных метода ТГц импульсной спектроскопии для численного анализа результатов. С учетом всех особенностей схемы измерения $[27,28]$ следует ограничиться частотой около $10 \mathrm{~cm}^{-1}(300 \mathrm{GHz})$, ниже которой возможны существенные ошибки в установлении характера диэлектрической дисперсии вещества.

Нагрев образца приводит не только к количественным, но и к качественным изменениям в спектрах, что указывает на происходящие в нем структурные изменения. Для спектра, измеренного при температуре $400 \mathrm{~K}$, зарегистрированы линии в области $30-40 \mathrm{~cm}^{-1}$, не характерные для $\alpha$-LM. Можно предположить, что появление этих линий связано с дегидратацией образца и образованием безводной формы $\beta$-лактозы [20]. Температура плавления лактозы составляет $202.8^{\circ} \mathrm{C}(476 \mathrm{~K})$. Наши измерения проведены в диапазоне до $475 \mathrm{~K}$, что немного ниже температуры плавления. Спектр пропускания для максимальной температуры лишен каких-либо резонансных особенностей, но имеет широкие диффузные полосы. Важным результатом измерений является сохранение слабо выраженной интерференционной картины на низких частотах. Это свидетельствует о том, что образец находится в твердой фазе, его форма - плоскопараллельная пластина - практически не искажена, при этом на низких частотах не появилось значительного поглощения.

Общий вид температурной эволюции диэлектрического отклика представлен в виде спектров действительной части динамической проводимости $\sigma^{\prime}(v)=2 \pi v \varepsilon_{0} \varepsilon^{\prime \prime}(v)$, где $\varepsilon_{0}=8.854 \cdot 10^{-12} \mathrm{~F} / \mathrm{m}$ - электрическая постоянная. На рис. 4 прослеживаются основные изменения в ТГц диэлектрическом отклике $\alpha$-LM во всем температурном диапазоне существования твердого образца. При криогенных температурах две полосы выше $60 \mathrm{~cm}^{-1}$ разделяются на высокодобротные линии; более того, проявляют- ся линии в областях 30 и $50-55 \mathrm{~cm}^{-1}$. Слабый резонанс около $30 \mathrm{~cm}^{-1}$ может быть связан с малым содержанием $\alpha$-формы лактозы. В области температур $350-450 \mathrm{~K}$ этот пик заметно увеличивается и уширяется. Однозначно идентифицировать линии поглощения в области $50-55 \mathrm{~cm}^{-1}$ сложно. Кардинальные изменения спектров вблизи температуры плавления $460-475 \mathrm{~K}$ могут быть связаны, с одной стороны, с ростом квазирелаксационных процессов и ростом констант затухания вблизи температуры фазового перехода и, с другой стороны, со структурными изменениями на молекулярном уровне.

Спектры пропускания образца после его охлаждения в вакуумной камере показаны точками на рис. 5, a. Модельный спектр пропускания на этом же графике показан сплошной линией, при этом достоверный расчет ограничен диапазоном $10-65 \mathrm{~cm}^{-1}$. Для того чтобы получить правильное описание дисперсии в области ниже $65 \mathrm{~cm}^{-1}$, мы использовали при моделировании дополнительный осциллятор, показанный пунктирной линией. Очевидно наличие полосы поглощения в области $60-65 \mathrm{~cm}^{-1}$, однако ее параметры можно оценить, учитывая только дисперсию низкочастотного крыла контура поглощения, причем с невысокой точностью. На более высоких частотах образец остается непрозрачным. На рис. 5, $b$ сплошной толстой линией показан спектр оптической проводимости. Для сравнения приведены спектр для температуры $310 \mathrm{~K}$ до отжига, показанный штрихпунктирной линией, и спектр при максимальной температуре (тонкая сплошная линия).

Из полученных спектров очевидно, что в процессе нагрева в образце произошли необратимые структурные изменения. Для того чтобы выяснить, связаны ли они с разделением лактозы на моносахариды, проведены аналогичные измерения образца глюкозы. В спектре глюкозы, показанном на рис. 5, $b$ точками, зарегистрированы дублеты в области 50 и $60-70 \mathrm{~cm}^{-1}$. Эти линии 
были идентифицированы при комнатной температуре для D- и L-форм глюкозы в работе [29].

Спектр охлажденного образца не содержит особенностей, присущих отклику глюкозы. Он характеризуется существенно большим поглощением и размытостью полос. Такой тип диэлектрического отклика характерен для разупорядоченных систем. В них за счет структурного беспорядка времена жизни колебаний существенно уменьшаются, что соответствует росту констант затухания. Вторым аргументом в пользу сильного разупорядочения стал широкий квазирелаксационный фон. Таким образом, нагрев $\alpha$-LM привел к необратимым изменениям структуры, происшедшим до плавления образца.

\section{Заключение}

Выполнены измерения спектров диэлектрического отклика $\alpha$-LM в ТГц области спектра в широком диапазоне температур. Показано, что полосы поглощения выше $50 \mathrm{~cm}^{-1}$ формируются перекрывающимися линиями температурно уширенных резонансов и могут быть разрешены при низких температурах. Собственные частоты всех этих полос демонстрируют заметно выраженную температурную зависимость. Важным фактором является необратимое исчезновение характерных полос при температурах выше $\sim 400 \mathrm{~K}$, что указывает на структурные изменения в образце. Таким образом, можно утверждать, что устойчивость $\alpha$-LM ограничена температурой порядка $400 \mathrm{~K}$, что ниже температуры плавления образца.

\section{Финансирование работы}

Анализ температурных зависимостей диэлектрического отклика поддержан РНФ 18-12-00328. Проведение измерений поддержано РФФИ № 19-32-50075 мол_нр.

\section{Конфликт интересов}

Авторы заявляют, что у них нет конфликта интересов.

\section{Список литературы}

[1] Shen Y-Ch., Taday P.F. // IEEE J. Selected Topics in Quantum Electronics. 2008. V. 14. N 2. P. 407. doi 10.1109/JSTQE.2007.911309

[2] Taday P.F., Bradley I.V., Arnone D.D., Pepper M. // J. Pharmaceutical Sciences. 2003. V. 92. N 4. P. 831. doi 10.1002/jps.10358

[3] Smolyanskaya O.A., Chernomyrdin N.V., Konovko A.A., Zaytsev K.I., Ozheredov I.A., Cherkasova O.P., Nazarov M.M., Guillet J.-P., Kozlov S.A., Kistenev Yu.V., Coutaz J.-L., Mounaix P., Vaks V.L., Son J.-H., Cheon H., Wallace V.P., Feldman Yu., Popov I., Yaroslavsky A.N., Shkurinov A.P., Tuchina V.V. // Progress in Quantum Electronics. 2018. V. 62. P. 1. doi 10.1016/j.pquantelec.2018.10.001
[4] Zaytsev K.I., Dolganova I.N., Chernomyrdin N.V., Katyba G.M., Gavdush A.A., Cherkasova O.P., Komandin G.A., Shchedrina M.A., Khodan A.N., Ponomarev D.S., Reshetov I.V., Karasik V.E., Skorobogatiy M., Kurlov V.N., Tuchin V.V. // J. Optics. 2020. V. 22. N 1. P. 013001. doi 10.1088/2040-8986/ab4dc3

[5] Lavrukhin D.V., Yachmenev A.E., Pavlov A.Yu., Khabibullin R.A., Goncharov Yu.G., Spektor I.E., Komandin G.A., Yurchenko S.O., Chernomyrdin N.V., Zaytsev K.I., Ponomarev D.S. // Semiconductor Science \& Technology. 2019. V. 34. P. 034005 . doi 10.1088/1361-6641/aaff31

[6] Komandin G., Anzin V., Ulitko V., Gavdush A., Mukhin A., Goncharov Y., Porodinkov O., Spektor I. // Optical Engineering. 2019. V. 59. N 6. P. 061603. doi 10.1117/1.OE.59.6.061603

[7] Zeitler J.A., Taday P.F., Newnham D.A., Pepper M., Gordon K.C., Rades T. // J. Pharmacy and Pharmacology. 2007. V. 59. N 2. P. 209. doi 10.1211/jpp.59.2.0008

[8] Markl D., Ruggiero M.T., Zeitler J.A. // European Pharmaceutical Review. 2016. V. 21. N 4. P. 45.

[9] Musina G., Dolganova I., Malakhov K., Gavdush A., Chernomyrdin N., Tuchina D., Komandin G., Chuchupal S., Cherkasova O., Zaytsev K., Tuchin V. // Proceedings of SPIE. Berlin, Germany, 2018. V. 10800. P. 108000F. doi $10.1117 / 12.2324473$

[10] Musina G.R., Gavdush A.A., Tuchina D.K., Dolganova I.N., Komandin G.A., Chuchupal S.V., Smolyanskaya O.A., Cherkasova O.P., Zaytsev K.I., Tuchin V.V. // Proceedings of SPIE. Berlin, Germany, 2019. V. 11065. P. $110651 Z$. doi $10.1117 / 12.2526168$

[11] Zeitler J.A., Kogermann K., Rantanen J., Rades T., Taday P.F., Pepper M., Aaltonen J., Strachan C.J. // Int. J. Pharmaceutics. 2007. V. 334. P. 78. doi 10.1016/j.ijpharm.2006.10.027

[12] Parimaladevi P., Srinivasan K. // Int. Dairy J. 2014. V. 39. P. 301. doi 10.1016/j.idairyj.2014.08.007

[13] Brown E.R., Zhang $W$-D. The Critical Effect of Hydratation on the Resonant Signatures of THz Biospectroscopy. [Электронный ресурс] Режим доступа: https://arxiv.org/abs/1810.08877

[14] Lowe E.K., Paterson A.H.J. // J. Food Engineering. 1998. V. 38. P. 15. doi 10.1016/S0260-8774(98)00109-5

[15] Komandin G.A., Nozdrin V.S., Gavdush A.A., Pronin A.A., Porodinkov O.E., Spektor I.E., Sigaev V.N., Mikhailov A.A., Shakhgildyan G.Yu., Ulitko V.E., Abdullaev D.A. // J. Appl. Phys. 2019. V. 126. P. 224303. doi 10.1063/1.5116790

[16] Yan S., Zhang H., Yang Z., Tang M., Zhang M., Du C., Cui H-L., Wei D. // RCS Adv. 2017. V. 7. N 66. P. 41667. doi 10.1039/c7ra07118c

[17] Walter M., Plochocka P., Fisher B., Helm H., UhdJepsen P. // Biopolimers (Biospectroscopy). 2002. V. 67. P. 310. doi 10.1002/bip.10106

[18] Beevers E.A., Hansen H.N. // Acta Crystallographica. Section B. 1971. V. 27. P. 1323. doi 10.1107/S0567740871003947

[19] Noordik J.N., Beurskens P.T., Bennema P., Visser R.A., Gould R.O. // Zeitschrift fur Kristallographie. 1984. V. 168. N 1-4. P. 59. doi 10.1524/zkri.1984.168.14.59

[20] Yamauchi S., Hatakeyam S., Imai Y., Tonouchi M. // American J. Analytical Chemistry. 2013. V. 4. N 12. P. 756. doi 10.4236/ajac.2013.412092

[21] Saito S., Inerbaev T.M., Mizuseki H., Igarashi N., Note R., Kawazoe Y. // Japan. J. Applied Physics. 2006. V. 45. N. 43. P. L1156. doi 10.1143/JJAP.45.L1156 
[22] Komandin G.A., Gavdush A.A., Goncharov Yu.G., Porodinkov O.E., Nozdrin V.S., Chuchupal S.V., Spektor I.E. // Opt. Spectrosc. 2019. V. 126. N 5. P. 514. doi 10.1134/S0030400X1905014X

[23] Komandin G.A., Chuchupal S.V., Lebedev S.P., Goncharov Yu.G., Korolev A.F., Porodoinkov O.E., Spektor I.E., Volkov A.A. // IEEE Transaction on Terahertz Science \& Technology. 2013. V. 3. N. 4. P. 440. doi 10.1109/TTHZ.2013.2255914

[24] Shen Y-C., Taday P.F. // IEEE J. Selected Topics in Quantum Electronics. 2008. V. 14. N 2. P. 407. doi 10.1109/JSTQE.2007.911309

[25] Roggenbuck A., Schmitz H., Deninger A., CamaraMayorga I., Hemberger J., Güsten G., Grüninger M. // New J. Physics. 2010. V. 12. P. 043017. doi 10.1088/1367-2630/12/4/043017

[26] Barker A.S., Hopfield J.J. // Phys. Rev. 1964. V. 135. P. A1732. doi 10.1103/PhysRev.135.A1732

[27] Guliano B.M., Gavdush A.A., Muller B., Zaitsev K.I., Gassi T., Palumbo M.E., Baratta G.A., Scire S., Komandin G.A., Yurchenko S.O., Casseli P. // Astronomy \& Astrophysics. 2019. V. 629. P. A112. doi 10.1051/0004-6361/201935619

[28] Gavdush A.A., Chernomyrdin N.V., Malakhov K.M., Beshplav S.-I.T., Dolganova I.N., Kosyrkova A.V., Nikitin P.V., Musina G.R., Katyba G.M., Reshetov I.V., Cherkasova O.P., Komandin G.A., Karasik V.E., Potapov A.A., Tuchin V.V., Zaytsev K.I. // J. Biomedical Optics. 2019. V. 24. N. 2. P. 027001. doi 10.1117/1.JBO.24.2.027001

[29] Upadhya P.C., Shen Y.C., Davies A.G., Linfield E.H. // J. Biological Physics. 2003. V. 29. N 2-3. P. 117. doi 10.1023/A:1024476322147 